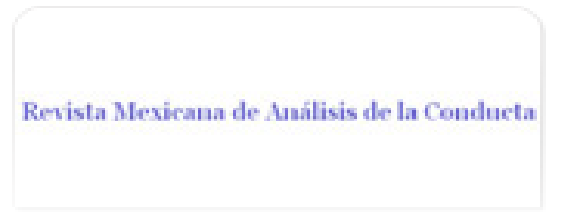

Revista Mexicana de Análisis de la Conducta ISSN: 0185-4534

editora@rmac-mx.org

Sociedad Mexicana de Análisis de la Conducta México

Cedillo-Ildefonso, B.; Arriaga, J.C.P.; Cruz-Morales, S. E.

Efectos del contexto en la tolerancia cruzada Diazepam-Etanol en el laberinto elevado en cruz (LEC)

Revista Mexicana de Análisis de la Conducta, vol. 34, núm. 1, enero-junio, 2008, pp. 111-129

Sociedad Mexicana de Análisis de la Conducta

Guadalajara, México

Disponible en: http://www.redalyc.org/articulo.oa?id=59311114008

Cómo citar el artículo

- Número completo

- Más información del artículo

- Página de la revista en redalyc.org

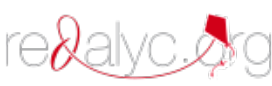

Sistema de Información Científica

Red de Revistas Científicas de América Latina, el Caribe, España y Portugal Proyecto académico sin fines de lucro, desarrollado bajo la iniciativa de acceso abierto 


\title{
EFECTOS DEL CONTEXTO EN LA TOLERANCIA CRUZADA DIAZEPAM-ETANOL EN EL LABERINTO ELEVADO EN CRUZ (LEC)
}

\author{
EFFECTS OF CONTEXT IN CROSSED TOLERANCE DIAZEPAM- \\ ETHANOL IN THE ELEVATED PLUS-MAZE (ETM)
}

\author{
B. CEDILLO-ILDEFONSO, J.C.P. ARRIAGA, Y S. E. CRUZ-MORALES' \\ PSICOFARMACOLOGÍA. UNAM \\ FES- IZTACALA, PSICOFARMACOLOGÍA.
}

\begin{abstract}
RESUMEN
La tolerancia es mayor cuando una droga es administrada en el contexto que señala la presencia de la droga. Este estudio fue diseñado para evaluar el efecto del contexto sobre el desarrollo de tolerancia al diazepam y de tolerancia cruzada diazepam-etanol, sobre la actividad exploratoria en el laberinto elevado en cruz (LEC) y sobre la actividad motora. Ratas Wistar machos fueron asignadas a grupos independientes $(n=8)$. En el Experimento 1 se evaluó el efecto de la administración aguda del etanol $(1.5 \mathrm{mg} / \mathrm{kg})$ y del diazepam $(1 \mathrm{mg} / \mathrm{kg})$. En el Experimento 2, se evaluó el efecto del contexto en el desarrollo de la tolerancia al diazepam: dos grupos crónicos recibieron 20 administraciones de diazepam $(1 \mathrm{mg} / \mathrm{kg})$ en diferentes contextos, uno en el contexto Bioterio y otro en el contexto Laboratorio, el grupo Control recibió 21 administraciones de salina en un volumen equivalente $(1 \mathrm{ml} / \mathrm{kg})$ en el contexto Laboratorio; el día de la prueba todos los grupos fueron evaluados en el contexto Laboratorio en el LEC. En el Experimento 3, siguiendo el mismo procedimiento se evaluó el efecto del contexto sobre la tolerancia cruzada diazepam-etanol. Inmediatamente después de la exposición al LEC, la actividad motora fue evaluada en todos los grupos. El diazepam y el etanol produjeron ansiólisis (Experimento 1), los grupos crónicos desarrollaron tolerancia,
\end{abstract}

1. Dirigir correspondencia a: Dra. Sara E. Cruz Morales. Apdo. Postal 314, Tlalnepantla, Edo. de México. 54090, México. 
independientemente del contexto, aunque fue mayor en el contexto asociado con la droga (Experimento 2). En el Experimento 3, se observó tolerancia cruzada diazepam-etanol y el efecto del contexto.

Palabras clave: diazepam, tolerancia cruzada, ansiedad, laberinto elevado en cruz, contexto

\begin{abstract}
Tolerance is stronger when the drug is administrated in the context that signals the presence of the drug. This study was designed to evaluate the effect of context on development of tolerance to diazepam and cross tolerance diazepam-ethanol, on exploratory activity in the elevated plus maze (EPM) and in motor activity. Male Wistar rats were assigned to independent groups ( $n$ $=8)$. In Experiment 1, the effect of acute administration of ethanol $(1.5 \mathrm{mg} / \mathrm{kg})$ and diazepam $(1 \mathrm{mg} / \mathrm{kg})$ was evaluated. In Experiment 2, the effect of context was evaluated on the development of tolerance: two chronic groups received 20 administrations of diazepam in different contexts, one in the Colony Room context and other in the Laboratory context, the Control group received 21 administration of saline in equivalent volume $(1 \mathrm{ml} / \mathrm{kg})$ in the Laboratory context; on test day all groups were evaluated in the Laboratory context. In Experiment 3 , following the same procedure, the effect of context was evaluated on the development of cross tolerance diazepam-ethanol. Immediately after exposure on the ETM, motor activity was evaluated in all groups. Diazepam and ethanol produced anxiolysis (Experiment 1); the chronic groups developed tolerance independently of the context, although it was stronger in the context associated with the drug (Experiment 2). In Experiment 3, the development of cross-tolerance diazepam-ethanol and the effect of context were observed.
\end{abstract}

Kew words: diazepam, cross tolerance, anxiety, elevated plus-maze, context.

La tolerancia es la disminución de los efectos a un fármaco por su aplicación repetida, por lo que se requiere de una dosis mayor a la administrada inicialmente para reinstalar los efectos iniciales del fármaco (O'Brien, 1996). La tolerancia a los diferentes efectos de las drogas se desarrolla a diferente razón, algunos efectos desarrollan tolerancia rápidamente, otros efectos desarrollan una lenta tolerancia, y en otros casos no se desarrolla tolerancia a algunos de los efectos (File, 1985).

La tolerancia tiende a desaparecer con el paso del tiempo después de que el uso del fármaco es descontinuado (McKim, 1986). Es importante se- 
ñalar que la tolerancia no solo se puede explicar farmacológicamente sino también en forma conductual, donde el aprendizaje es una variable importante para la presencia o ausencia de este fenómeno

Uno de los procesos de aprendizaje que explica el desarrollo de la tolerancia está basado en el modelo de condicionamiento clásico. Pavlov (1927) administró pequeñas dosis de apomorfina, estímulo incondicionado (EI), que inducía la respuesta incondicionada (RI) de vómito, 1 ó 2 minutos después se presentaba un tono (estímulo neutro, EN) que se mantenía mientras iniciaba la respuesta a la droga, después de varios ensayos, el EN adquiría funciones de El que inducía por si solo la respuesta de vómito, considerada como respuesta condicionada (RC). Se ha observado que cuando un usuario de drogas está ante los estímulos (condicionados) asociados previamente a la administración de la droga, se observa una reducción de sus efectos, desarrollándose la tolerancia (Siegel, 1985). Siegel (1985) sugirió que como consecuencia de las administraciones repetidas del fármaco se producen respuestas compensatorias condicionadas (RCC) que en general son opuestas al efecto original del fármaco, y que los estímulos asociados a la administración del fármaco podrían modular el desarrollo de la tolerancia; para estudiar la participación de los estímulos condicionados y el papel de las respuestas compensatorias en el desarrollo de la tolerancia realizó diversos estudios. En uno de estos estudios asoció la administración de morfina en dos situaciones diferentes y observó que cuando los sujetos eran evaluados en la misma situación había una disminución de la respuesta, es decir, se desarrollaba tolerancia y no se detectaba el efecto analgésico; mientras que cuando la administración de morfina se hacía en una situación diferente los sujetos presentaban hiperalgesia (Siegel, 1975). De acuerdo a Siegel (1975), la tolerancia es menor si la persona se administra la droga en ambientes novedosos, porque las nuevas claves o estímulos condicionados no provocan la respuesta compensatoria.

La presencia de las respuestas compensatorias se ha reportado en diferentes situaciones (Greeley \& Cappell, 1985; Poulos, Hunt \& Cappell, 1988; Siegel, 1983), sin embargo no siempre se detectan. Por ejemplo, CepedaBenito, Tiffany y Cox (1999) evaluaron la tolerancia asociativa a los efectos analgésicos de la morfina en dos pruebas, en la primera la respuesta era retirar la pata como consecuencia de aplicar presión y en la otra se midió el umbral de vocalización de la rata cuando se aplicaba un choque en la cola. Se dieron tratamientos crónicos con morfina apareada a un contexto distintivo o en la caja-hogar, posteriormente los sujetos se evaluaron con morfina o placebo en el contexto distintivo. Los resultados demostraron que la tolerancia fue específica al contexto con ambas pruebas, pero no se evidenció la respuesta compensatoria en ningún caso. Cabe señalar que en humanos, los estímulos asociados al consumo de una droga también provocan sensación 
de abstinencia y ansiedad por consumir la droga; e incluso después de haber pasado el síndrome de abstinencia a la droga, se presentan recaídas, y aparentemente muchas de las recaídas son provocadas por el contacto del organismo con los estímulos asociados al consumo (Siegel \& Ramos, 2002).

A este tipo de tolerancia se le llama tolerancia asociativa o dependiente del contexto y se detecta cuando la droga es administrada por la misma vía de administración, en presencia de las mismas señales asociadas a la administración y en el mismo contexto en que se hace la prueba de tolerancia (Siegel, 1989). Se ha observado que cuando se presenta la tolerancia asociativa, los estímulos asociados con la administración de la drogas son capaces de predecir la siguiente administración de la misma (Bell, Olson \& Vaccarino, 1998).

El uso crónico de las benzodiazepinas produce tolerancia y dependencia, fenómenos relacionados en forma importante con la farmacodinamia de las benzodiazepinas (File, 1985). Actualmente se sabe que se desarrolla tolerancia a los efectos sedantes, anticonvulsivantes, y miorrelajantes, mientras que el desarrollo de tolerancia a los efectos ansiolíticos es más lento (File, 1984; File, 1985; Treit, 1985a). En los estudios sobre el desarrollo de tolerancia al efecto ansiolítico de las benzodiazepinas, se ha detectado tolerancia empleando diferentes benzodiazepinas y diferentes modelos para medir ansiedad. Por ejemplo, en la prueba de interacción social se reportó que el clordiazepóxido pierde parte de su acción ansiolítica después de 15 y 25 administraciones (Vellucci \& File, 1979) y en el laberinto elevado en cruz (LEC) después de 20 administraciones (File, Baldwin \& Aranko (1987); el mismo efecto de tolerancia se observa después de 9 días de administraciones de $5 \mathrm{mg} / \mathrm{kg}$, po de diazepam en una prueba de castigo (Stephens \& Schneider, 1985), y con 8 días de tratamiento con diazepam se observa tolerancia a los efectos ansiolíticos en ratones con experiencia previa en el LEC (Rodgers \& Shepherd, 1993). Asimismo, Fernández y File (1999) reportan el desarrollo de tolerancia a los efectos ansiolíticos del diazepam (2 mg/kg, ip) en un a prueba de interacción social después de 21 días. Begg, Hallam y Norman (2005) obtuvieron tolerancia al diazepam (4 mg/kg, ip) con administraciones de 14 días en pruebas de interacción social y en el LEC. En tanto que Schmitt, Luudens y Hiemke (2001) reportaron tolerancia al diazepam $(10 \mathrm{mg} / \mathrm{kg}$, ip) después de 23 días de tratamiento en una prueba en el LEC.

Sin embargo no siempre se encuentra tolerancia, por ejemplo la administración de $20 \mathrm{mg} / \mathrm{kg}$, ip de oxazepam por 22 días no produjo tolerancia en una prueba de castigo (Margules \& Stein, 1968). Con diazepam no se detectó tolerancia después de $1 \mathrm{mg} / \mathrm{kg}$, ip de diazepam por 9 días en la prueba de interacción social (de Angelis \& File, 1979), con $5 \mathrm{mg} / \mathrm{kg}$, ip por 14 días en el LEC (Harro, Lang \& Vasar, 1990) o en la prueba de sobresalto (Hijzen, Woudenberg \& Slangen, 1990). 
Como puede observarse, los resultados sobre el desarrollo de la tolerancia por las benzodiazepinas no son consistentes ya que estos dependen del tipo de benzodiazepina y por tanto de su vida media, del tipo de tratamiento y de la prueba. Por ejemplo, las benzodiazepinas de acción corta como el midazolam desarrollan más rápidamente tolerancia (Irvine, Cheeta, Lovelock \& File, 2001) que las de acción larga como el diazepam (Fernández \& File, 1999).

Se ha reportado que la tolerancia a un efecto particular también produce tolerancia cruzada a otras drogas que tienen el mismo efecto farmacológico que la primera, esto es, el organismo responde menos a los efectos de una droga como consecuencia de tratamientos previos con otras drogas, la tolerancia cruzada se presenta con drogas de la misma clase o que tienen efectos similares (Le, Khanna \& Kalant, 1987; Thompson \& Boren 1983). Por ejemplo, los tratamientos crónicos con etanol inducen tolerancia cruzada a varias benzodiazepinas y barbitúricos (Khanna, Kalant, Weiner \& Shah, 1992).

La importancia del contexto no solo se manifiesta en el desarrollo de la tolerancia a las drogas, sino también en el desarrollo de la tolerancia cruzada (Khanna, et al., 1992). De acuerdo con la propuesta de Siegel (1989), en el caso de la tolerancia cruzada, si ambas drogas tienen efectos similares, la respuesta a la primera droga compensa sus efectos y entonces la respuesta compensatoria a la droga 2 será atenuada en presencia de las señales o contexto de la droga 1, produciéndose de esta forma la tolerancia cruzada.

De acuerdo a los planteamientos asociativos, parece que los estímulos condicionados a las drogas tienen un papel relevante en los fenómenos de tolerancia, abstinencia y en las recaídas. Esto sugiere que el debilitamiento de la asociación entre la droga y los estímulos relacionados con su consumo juegue un papel importante en el tratamiento de las adicciones. A pesar de la evidencia en la literatura de los mecanismos asociativos en el desarrollo de tolerancia a diferentes drogas, existe poca evidencia sobre los mecanismos de condicionamiento en el desarrollo de la tolerancia a las benzodiazepinas (File, 1982; Greeley \& Cappell, 1985; King, Bouton \& Musty, 1987).

Esta investigación estudió el efecto del contexto en el desarrollo de la tolerancia cruzada condicionada del diazepam al etanol, evaluada en la actividad exploratoria en el laberinto elevado en cruz. En todos los casos se siguió el mismo procedimiento, los sujetos fueron sometidos a los diferentes tratamientos farmacológicos antes de la exposición en el LEC. En el Experimento 1 se evaluó el efecto de la administración aguda de diazepam y etanol para demostrar que ambos compuestos producen un efecto ansiolítico; en el Experimento 2 se evalúo el efecto del contexto sobre el desarrollo de tolerancia al diazepam administrado crónicamente; y en el Experimento 3 se evaluó el efecto del contexto sobre la tolerancia cruzada diazepam-etanol. Con la 
finalidad de descartar que los efectos obtenidos pudieran deberse a cambios inducidos por alteraciones en la actividad motora y no al efecto ansiolítico, se realizaron pruebas adicionales donde se evaluó la actividad motora, inmediatamente después de la prueba en el LEC.

\section{MÉTODO GENERAL}

\section{Sujetos}

Se utilizaron ratas macho Wistar, experimentalmente ingenuas, de aproximadamente $200 \mathrm{~g}$ de peso al inicio del experimento, provenientes del Bioterio general de la FES Iztacala-UNAM. Los sujetos (Ss) fueron alojados en cajas de acrílico transparente ( 5 sujetos por caja), bajo un ciclo de luz/obscuridad $12: 12$, con libre acceso a agua y comida.

\section{Aparatos}

Laberinto elevado en cruz (LEC): El LEC está construido de PVC con dos brazos abiertos $(50 \times 10 \mathrm{~cm})$, y dos brazos cerrados de la misma medida, elevado a $50 \mathrm{~cm}$ del piso, el nivel de iluminación fue de 50 lux. El diseño del laberinto se conforma de tal forma que los mismos tipos de brazos son opuestos a los otros.

Cámara de vídeo grabación: Se empleó una cámara (CCD, Modelo CA156SEIA) localizada $1 \mathrm{~m}$ arriba del cuadro central del laberinto, lo cual permitía una visión de los 4 brazos del laberinto. Los registros fueron grabados (Videograbadora WJ-2096) y analizados posteriormente con el Software Observer (Universidad de Sao Paulo, Brazil).

Cámara de actividad motora: Una cámara construida de acrílico negro

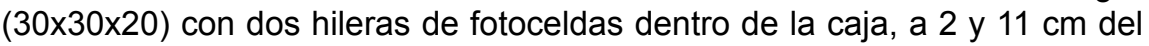
piso, y con $2 \mathrm{~cm}$ de distancia entre las fotoceldas. La hilera de fotoceldas más cercana al piso detecta la actividad horizontal (deambulación) y la hilera alta detecta la actividad vertical (alzadas) en cada rata.

\section{Drogas}

Se administró intraperitonealmente (ip) diazepam (Roche, México, D. F) diluido en solución salina $(0.9 \%)$, en una dosis de $1 \mathrm{mg} / \mathrm{kg}$. Esta dosis se determinó con base en una curva dosis-respuesta, donde con $1 \mathrm{mg} / \mathrm{kg}$ de diazepam se observó mejor el efecto ansiolítico, sin afectar la actividad motora, dado que el efecto de sedación se observó a dosis más altas. Dosis equivalentes se han empleado en investigaciones previas, donde con $1 \mathrm{mg} / \mathrm{kg}$ de diazepam se observó el desarrollo de tolerancia al efecto anticonvulsivo (Kalynchuk, Kim, Pinel \& Kippin, 1994). En el caso del etanol se ha reportado que 
$1.5 \mathrm{~g} / \mathrm{kg}$ en una solución al $20 \%$ produce el efecto ansiolítico (Bertoglio \& Carobrez, 2002).

\section{PROCEDIMIENTO}

LEC: Se grabó la sesión para posteriormente registrar los parámetros conductuales que son la frecuencia del número de entradas a los brazos abiertos y cerrados, así como la cantidad del tiempo de permanencia en estos y en el cuadro central para cada uno de los sujetos. El criterio en ambos casos era que los sujetos tuvieran las 4 patas en el brazo; la duración de la sesión fue de cinco minutos. Estos datos se usaron para calcular el porcentaje de entradas a los brazos abiertos (EBA) de acuerdo a la siguiente fórmula: [(No. entradas a brazos abiertos/ (No. abiertos +cerrados)) x 100] y el porcentaje de tiempo de permanencia en brazos abiertos (TBA): [(tiempo en brazo abierto/300) $\mathrm{x} 100$ )], así como el total de entradas (entradas en brazos abierto+entradas en brazos cerrados). Las pruebas fueron grabadas por la cámara de video y posteriormente fueron analizadas.

Actividad Motora: Al término de las sesiones en el LEC, a los sujetos se les introdujo en la cámara de actividad motora durante 30 min y se registró el número de cruces a intervalos de 5 min. El número de total de cruces se imprimió para un análisis posterior de la actividad vertical y horizontal.

Análisis de datos

Los datos fueron analizados mediante ANOVAS de una vía con el tratamiento como factor para la respuesta de ansiedad evaluada por el porcentaje de entradas y de permanencia en los brazos abiertos. Cuando se detectaron diferencias significativas se aplicó la prueba estadística de Tukey para diferencias significativas entre pares de grupos. Se realizaron análisis independientes para la actividad motora horizontal y vertical por medio de ANOVAS de medidas repetidas, para detectar las posibles diferencias por tratamientos, seguidos por la prueba de Tukey.

\section{EXPERIMENTO 1. EFECTO DEL DIAZEPAM Y EL ETANOL EN EL LEC}

El etanol es una droga de las más consumidas entre la población y uno de sus efectos es ansiolítico al igual que el diazepam a dosis bajas. Por otro lado, se ha descrito que la tolerancia cruzada se presenta a otra droga que tiene efectos similares (diazepam-etanol). Por lo cual el objetivo de este experimento, fue estudiar el efecto de la administración aguda de diazepam y 
etanol con la finalidad de demostrar que ambos compuestos producen efectos ansiolíticos comparables evaluados en el LEC.

\section{Procedimiento}

Los Ss fueron asignados aleatoriamente a tres grupos independientes $(n=8)$ que recibieron uno de los siguientes tratamientos: diazepam (D), etanol (E) o solución salina (S) en un volumen equivalente de $1 \mathrm{ml} / \mathrm{kg}$. Cada rata fue colocada en el cuadro central viendo hacia el brazo cerrado del LEC 30 min después de la administración de la droga correspondiente durante 5 min para su libre exploración en este aparato; al término de la exposición en el LEC, se evalúo la actividad motora. El laberinto y la cámara de actividad motora, se limpiaban entre cada sujeto con una franela con alcohol.

\section{Resultados y Discusión}

La Figura 1 muestra que los promedios del porcentaje de entradas a los brazos abiertos (EBA) se encontraron diferencias estadísticamente significativas $(F(2,21)=10.47 ; p<.001)$. Comparaciones entre grupos mostraron diferencias significativas de los grupos de $E$ y $D$ respecto al grupo $S(p<.001$ y $p<.05$ ) respectivamente, observándose el efecto ansiolítico tanto del etanol como del diazepam en el porcentaje de entradas a los brazos abiertos. No se detectaron diferencias entre los grupos de $\mathrm{D}$ y $\mathrm{E}$.

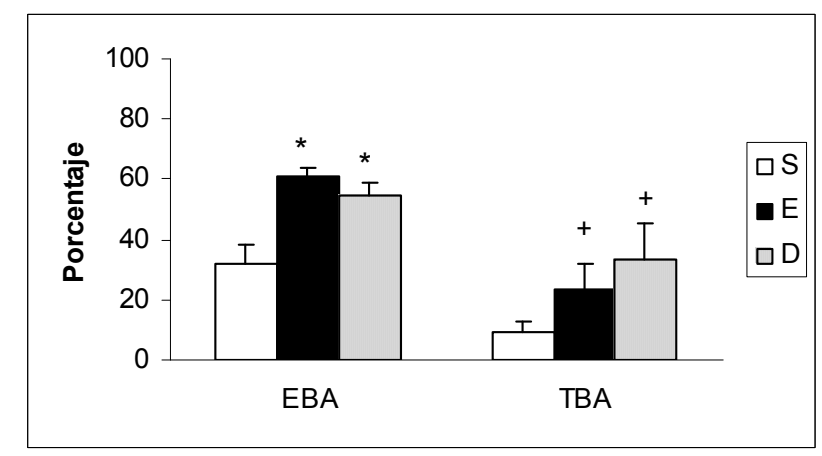

Figura 1. Efectos ansiolíticos de la administración aguda de etanol (1.5 $\mathrm{g} / \mathrm{kg})$ y diazepam $(1 \mathrm{mg} / \mathrm{kg})$ en ratas. Representa las medias \pm EEM del porcentaje de entradas en los brazos abiertos (EBA) y tiempo en los brazos cerrados (TBA); Salina (S), Etanol (E), Diazepam (D). ${ }^{* *} p<.001,{ }^{*} p<.05$ vs. $S ;+p<.05$ vs. $S$. 
En el porcentaje de tiempo de permanencia en los brazos abiertos (TBA), también se obtuvieron diferencias significativas $(F(2,21)=6.32, p<.05)$. En la Figura 1 se puede observar que los grupos de etanol y diazepam no difirieron entre ellos, pero difirieron con respecto al grupo salina $(p<.05)$.

En el número total de entradas no se obtuvieron diferencias significativas $(F(2,21)=3,33, p>.05)$, lo que indica que el tratamiento de etanol al igual que el diazepam no afectan la actividad motora. Estos datos coinciden con los datos obtenidos en la prueba de actividad motora, donde no se detectaron diferencias significativas en la interacción de los factores (tratamiento $\mathrm{x}$ tiempo) en la actividad horizontal $(F(10,105)=1.43, p>0.05)$ ni en la actividad vertical $(F(10,105)=1.30, p>.05)$.

En este experimento se demostraron los efectos ansiolíticos por la administración aguda de diazepam $(1 \mathrm{mg} / \mathrm{kg})$ y etanol $(1.5 \mathrm{~g} / \mathrm{kg})$. Los efectos ansiolíticos del etanol $(1.5 \mathrm{~g} / \mathrm{kg})$ observados en la prueba del LEC, coinciden con los trabajos reportados previamente por Bertoglio y Carobrez (2002). Los efectos ansiolíticos del diazepam han sido demostrados con la misma dosis en la prueba de enterramiento (Treit, 1985b), en pruebas de interacción social (de Angelis \& File, 1979) y con $5 \mathrm{mg}$ de diazepam en la misma prueba del LEC (Harro, et al., 1990). Es importante resaltar que con estas dosis de etanol y de diazepam en el presente experimento, no se afectó la actividad motora del animal, dado que no se encontraron cambios en el número total de entradas en el LEC lo cual fue corroborado en la prueba de actividad motora, donde los resultados no presentaron diferencias ni en la actividad motora vertical ni en la actividad motora horizontal. Los resultados de este experimento son importantes porque es necesario primero demostrar el efecto ansiolítico por la administración aguda para posteriormente poder estudiar el desarrollo de tolerancia por la administración crónica y la influencia del contexto.

\section{EXPERIMENTO 2. EFECTO DEL CONTEXTO EN EL DESARROLLO DE TOLERANCIA AL DIAZEPAM}

Algunos datos sugieren un mayor efecto del contexto en el aprendizaje cuando en lugar de presentar estímulos discretos aislados, se presenta el contexto como un compuesto de estímulos (configuración) observándose un mayor control de éste (Fantino, 2001; Rescorla \& Wagner, 1972). Existen datos que sugieren que los sujetos perciben el contexto asociado con las administraciones de la droga como estímulos discretos (Cox \& Tiffany, 1997) o bien en forma configuracional, de tal forma que los organismos perciben las características de los estímulos que integran el contexto como un compuesto y no en forma aislada (Caggiula, et al., 1991). Con base en lo anterior, se realizó el Experimento 2 con el objetivo de estudiar el efecto del contexto entendido 
como un compuesto de estímulos sobre el desarrollo de tolerancia al efecto ansiolítico del diazepam.

El contexto se refiere a aspectos o claves del ambiente o a estímulos que afectan el grado de condicionamiento de una respuesta (Fantino, 2001). En este experimento, el contexto se consideró como el espacio físico formado por todos los estímulos contenidos en el Bioterio o en el Laboratorio. La manipulación del contexto fue dada por el cambio del lugar físico donde se administró la droga (Laboratorio o Bioterio).

Bioterio. Este espacio contenía las jaulas donde se colocaron a las ratas, 2 estantes, una mesa, una balanza con la que se pesaba a los sujetos y tenía luz natural.

Laboratorio: En este espacio se encontraba el LEC, el equipo de vídeo grabación, un generador de ruido blanco, con iluminación de 50 lux.

\section{Procedimiento}

Tres grupos fueron tratados crónicamente: dos grupos fueron inyectados con $1 \mathrm{mg} / \mathrm{kg}$, ip de diazepam por 20 días (1 por día), el primer grupo de diazepam (D) fue inyectado en el Laboratorio y el segundo en el Bioterio (DB), un tercer grupo fue inyectado con salina $(S)$ en un volumen equivalente $(1 \mathrm{ml} / \mathrm{kg})$ en el Laboratorio; 30 min después de cada administración los sujetos inyectados en el Laboratorio fueron regresados a su caja de alojamiento.

\section{Prueba de tolerancia}

El día 21 a los grupos $D$ y DB se les administró diazepam $(1.0 \mathrm{mg} / \mathrm{kg}$, ip) y al tercer grupo $\mathrm{S}$ se le administró solución salina en un volumen equivalente, en todos los casos la administración se hizo en el contexto Laboratorio siguiendo el mismo procedimiento que en la fase anterior. Se colocó a cada sujeto en el LEC en el cuadro central durante 5 minutos, se grabó la sesión y posteriormente se registraron los diferentes parámetros conductuales. Al finalizar la prueba en el laberinto cada sujeto fue trasladado al cuarto donde se encontraba la caja de actividad motora y se colocó al sujeto dentro de ésta durante $30 \mathrm{~min}$, y se registró la actividad a intervalos de $5 \mathrm{~min}$.

\section{Resultados y Discusión}

Los ANOVAS no detectaron diferencias en el porcentaje de entradas a los brazos abiertos $(F(2,21)=2.39 ; p<.1161)$ ni en el porcentaje de tiempo de permanencia en los brazos abiertos $(F(2,21)=3.32 ; p<.0561)$. Como puede observarse en la Figura 2, el grupo DB que fue evaluado en un contexto diferente muestra valores más altos en ambas medidas, comparado con los grupos $S$ y $D$ lo que sugiere un efecto ansiolítico, sin embargo no se encontraron diferencias estadísticamente significativas, por lo que se puede concluir que 
en ambos casos se observó tolerancia al efecto ansiolítico independientemente del contexto donde se les evaluó.

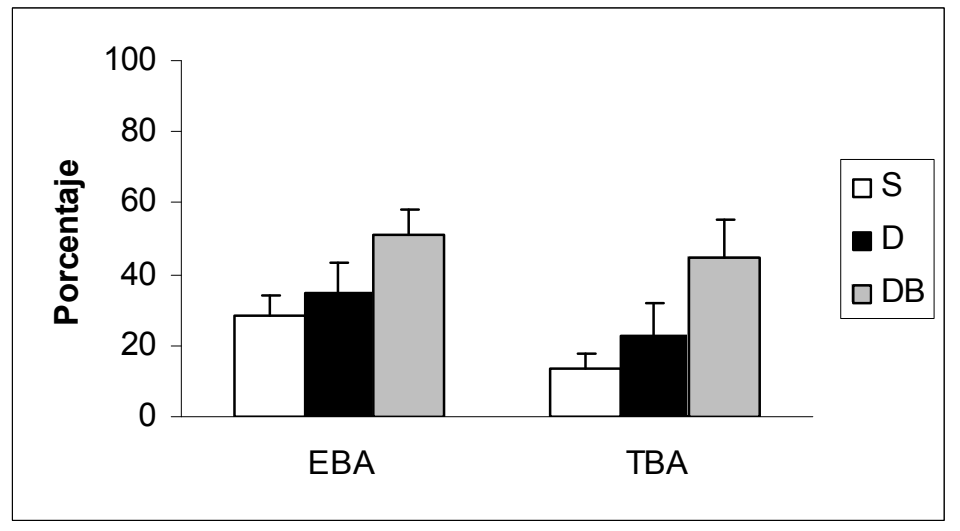

Figura 2. Efectos de la administración crónica de diazepam (1 mg/kg) en ratas evaluadas en el mismo contexto o en el contexto Bioterio. Representa las medias $\pm E E M$ del porcentaje de entradas en los brazos abiertos (EBA) y tiempo en los brazos abiertos (TBA); Salina (S), Diazepam (D), Bioterio (B).

No se obtuvieron diferencias significativas en el total de entradas, por lo que se descarta que el tratamiento altere la actividad motora de la rata.

Así mismo, en la prueba de actividad motora no se obtuvieron diferencias estadísticamente significativas al hacerse una prueba de medidas repetidas, esto es, no se mostró la interacción tratamiento/tiempo tanto en la actividad vertical como la actividad horizontal ( $p>.05$ ).

Los resultados de este experimento, demostraron el desarrollo de tolerancia al efecto ansiolítico del diazepam. Sin embargo, solo se detectó tolerancia farmacológica y no tolerancia asociativa. Esto es, no se demostró el efecto del contexto en el desarrollo de la tolerancia al efecto ansiolítico del diazepam con la dosis empleada. Sin embargo empleando la misma droga, nuestros resultados coinciden con el trabajo de Treit (1985b) quien obtuvo el desarrollo de tolerancia al efecto ansiolítico de $1 \mathrm{mg} / \mathrm{kg}$ de diazepam en la conducta defensiva de enterramiento después de 10 días de tratamiento, esta tolerancia fue independiente del contexto y dependiente de la intensidad del choque. Estos datos sugieren que probablemente las dosis bajas empleadas en ambos estudios son suficientes para producir tolerancia farmacológica, pero no tolerancia asociativa. En otros estudios se ha reportado mayor grado de tolerancia a los efectos atáxicos y sedantes, y poca o nula 
tolerancia a los efectos ansiolíticos de las benzodiazepinas. Por ejemplo, File (1985) observó mayor desarrollo de tolerancia a los efectos sedantes del lorazepam y triazolam que al efecto ansiolítico; asimismo los casos en que se ha descrito el desarrollo de tolerancia asociativa, este ha sido reportado para los efectos sedantes e hipotérmicos del clordiazepóxido (Greeley \& Cappell, 1985). Se ha descrito que la tolerancia a los efectos ansiolíticos (Pérez, Maglio, Marchesini, Molina \& Ramírez, 2002; Stephens \& Schneider, 1985), y al efecto hipolocomotor (Pérez et al., 2002) se presenta con dosis mayores de diazepam $(5 \mathrm{mg} / \mathrm{kg})$. Más aún, nuestros resultados son similares a lo reportado por Marin, Pérez, Duero y Ramírez (1999), quienes reportaron que la manipulación del contexto con la continua administración de diazepam tuvo poco efecto para mostrar la respuesta condicionada de la actividad locomotora y que probablemente se debió a que el tiempo de tratamiento fue corto (tres días).

De acuerdo a la propuesta de Baker y Tiffany(1985) la tolerancia asociativa se presenta con dosis bajas e intervalos entre administración largos, mientras que la farmacológica se presentaría con dosis grandes e intervalos cortos, sin embargo los hallazgos de Cepeda-Benito y Tiffany ponen en duda esta teoría, dado que estos autores reportan el desarrollo de tolerancia asociativa con dosis altas de morfina e intervalos de 96 horas y con dosis altas de morfina e intervalos de 6 horas se presenta tolerancia no asociativa (Cepeda-Benito \& Tiffany, 1996). En el presente experimento el intervalo entre administración fue de $24 \mathrm{hrs}$ y una dosis de $1 \mathrm{mg} / \mathrm{kg}$ del diazepam, y aunque al parecer el intervalo es largo, la dosis no permitió llevar a cabo la discriminación de las características de los estímulos, entre las asociaciones de las administraciones y condiciones de la prueba. Lo que hace suponer que el problema de la falta de tolerancia no solo podría deberse al contexto, si no que podría deberse a la dosis, y al intervalo entre administraciones.

\section{EXPERIMENTO 3. EFECTO DEL CONTEXTO SOBRE LA TOLERANCIA CRUZADA ETANOL DIAZEPAM}

La tolerancia cruzada puede ser demostrada por el decremento de los efectos de una droga, como consecuencia de la administración previa de otra droga, con estructura química, efectos, o mecanismos similares (O'Brien, 1996). Se ha obtenido tolerancia cruzada al efecto anticonvulsivante del flurazepam con diferentes benzodiazepinas (Rosenberg, 1995). Además de la tolerancia cruzada entre benzodiazepinas, también se ha reportado tolerancia cruzada entre etanol y benzodiazepinas. Por ejemplo, la administración de etanol por dos días produjo tolerancia cruzada al diazepam, clordiazepóxido, flurazepam, y oxazepam en la actividad motora medida en la prueba de plano incli- 
nado (Khanna, et al., 1992). Otros estudios han demostrado que la tolerancia cruzada etanol-clordiazepóxido, depende del tipo de prueba o respuesta empleada en la evaluación. Por ejemplo, se reportó tolerancia cruzada completa en la respuesta de hipotermia, tolerancia parcial en la viga horizontal y no se detectó tolerancia en la conducta de asomarse (Chan, Langan, Leong, Schanley \& Penetrante, 1988).

Existe información sobre tolerancia cruzada de las benzodiazepinas al etanol y del etanol a las benzodiazepinas en animales (Khanna, et al., 1992), sin embargo, no existen datos sobre el papel que los procesos asociativos tienen en el desarrollo de la tolerancia cruzada benzodiazepinas-etanol al efecto ansiolítico.

Aunque en los resultados del experimento anterior no se detectaron diferencias estadísticamente significativas, si se mostró una tendencia que sugiere que hay un efecto del contexto, dado que el grupo evaluado en un contexto diferente mostró un mayor porcentaje del número y del tiempo de permanencia en los brazos abiertos comparado con el grupo $S$ y el grupo evaluado en el mismo contexto. Por lo que el objetivo de este experimento, fue evaluar el papel del contexto en el desarrollo de la tolerancia cruzada diazepam-etanol al efecto ansiolítico, evaluado en el LEC.

\section{Procedimiento}

Se emplearon ratas macho tratadas bajo las mismas condiciones descritas en el método general, los sujetos fueron asignados al azar a tres grupos $(n=8)$. El primer grupo recibió diazepam durante 20 días en el contexto Laboratorio y el día 21 se realizó la prueba de tolerancia cruzada al etanol, se administró etanol ( $D+E)$; el segundo grupo recibió 20 administraciones de diazepam en el contexto Bioterio y el día 21 de la prueba fue inyectado con etanol en el Laboratorio (DB+E); el grupo de salina recibió 20 administraciones de solución salina en el contexto Laboratorio y el día 21 se le probó con salina en el Laboratorio, 30 min después de la administración del fármaco durante los 20 días se regresaron a sus cajas de alojamiento en el Bioterio, el día de la prueba se llevó a cada sujeto al LEC, donde fue probado durante 5 min.

Se siguió el mismo procedimiento de registro y análisis que en el experimento 1. Finalizada la prueba en el LEC, se llevó a cada sujeto a la cámara de actividad motora para la prueba de esta actividad con una duración de 30 min, al finalizar se regresaron a los sujetos al Bioterio.

\section{Resultados y Discusión}

La Figura 3 muestra que los resultados de este experimento revelaron diferencias significativas al aplicarse una ANOVA de una vía $F(2,21)=11.85 ; p<$ .001 , en el porcentaje de entradas a los brazos abiertos. 
En las comparaciones entre grupos no se obtuvieron diferencias significativas entre el grupo Salina y el grupo de administraciones crónicas de diazepam en el mismo contexto del Laboratorio (D+E: $p>.05$ ), lo que demuestra el desarrollo de tolerancia cruzada diazepam-etanol al efecto ansiolítico. Sin embargo, se detectaron diferencias estadísticamente significativas entre el grupo que recibió las administraciones crónicas de diazepam en el contexto Bioterio y que fue evaluado con etanol $(D B+E)$ y los grupos $S$ y $D+E$ que fueron tratados crónicamente y probados en el mismo contexto (Laboratorio) ( $p<$ .01 y $p<.001$ respectivamente), es decir, el grupo DB+E mostró ansiólisis.

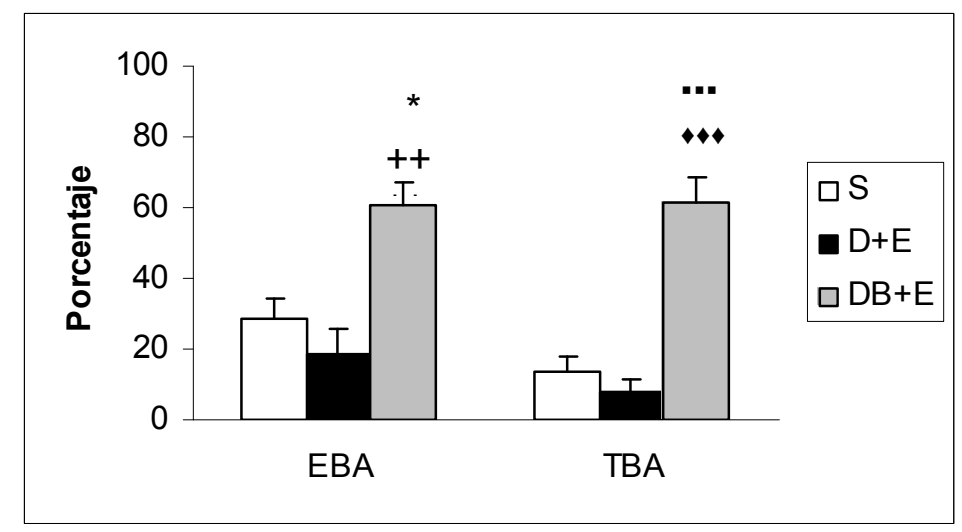

Figura 3. Tolerancia cruzada diazepam-etanol, en ratas pre-tratadas con diazepam y evaluadas con etanol en el mismo contexto $(D+E)$ o en ratas evaluadas en el contexto Bioterio $(D B+E)$. Representa las medias $\pm E E M$ del porcentaje del porcentaje de entradas en brazos abiertos (EBA) y el porcentaje de tiempo en los brazos abiertos (TBA), Salina (S). * $p<0.01 \mathrm{vs.}$ $S ;++p<0.001$ vs. $D+E ; \cdots p<0.0001$ vs. $S ; \leftrightarrow \bullet p<v s . D+E$

Efectos equivalentes se observaron en el porcentaje de tiempo de permanencia en los brazos abiertos. Un ANOVA mostró diferencias estadísticamente significativas, $F(2,21)=33.37, p<.0001$. No se encontraron diferencias significativas entre los grupos $S$ y $D+E$ evaluados en el mismo contexto donde recibieron las administraciones crónicas $(p>.05)$. El grupo evaluado en un contexto diferente al de sus administraciones previas (DB+E) difirió significativamente de los grupos $S$ y $D+E(p<.0001)$, es decir no se detectó tolerancia.

Respecto al total de entradas no se obtuvieron diferencias significativas $(p>.05)$ entre grupos, lo que indica que el tratamiento no afectó la actividad motora. En la actividad motora medida como actividad horizontal y vertical 
no se presentó la interacción entre los factores tratamiento/tiempo $(p>.05)$. El que no se detectaran diferencias en ambas medidas muestra que el tratamiento no afecta la actividad motora. Estos resultados demuestran el desarrollo de tolerancia cruzada diazepam-etanol al efecto ansiolítico y que este efecto es dependiente del contexto.

En este experimento se evalúo el efecto del contexto en el desarrollo de la tolerancia cruzada de las benzodiazepinas al etanol. Se demostró el desarrollo de tolerancia cruzada al etanol dependiente del contexto, tanto en el porcentaje del número de entradas como en el de permanencia en los brazos abiertos, donde el grupo pre-tratado y evaluado en el mismo contexto mostró un decremento en ambas medidas; mientras que el grupo pre-tratado y evaluado en un contexto diferente muestra un efecto ansiolítico en ambas medidas comparable al obtenido después de la administración de diazepam y etanol en el Experimento 1. Estos datos coinciden con el reporte de que la tolerancia cruzada midazolam-etanol en el LEC puede ser condicionada y por tanto dependiente del contexto (Cruz-Morales \& Cedillo-lldefonso, 2008).

Lo importante de estos resultados es que demostraron que el contexto puede modular la presencia y ausencia del desarrollo de la tolerancia, cuando las señales presentes durante las administraciones repetidas del diazepam, se presentan durante la prueba con etanol en el LEC, lo cual apoya los planteamientos de la relevancia de las señales asociadas con la administración de la droga (Le, Poulos \& Cappell, 1979; Greeley \& Cappell, 1985), ya que al parecer éstas facilitan la información adquirida en la asociación con el El (diazepam). En este caso, facilitaron el desarrollo de tolerancia cruzada al etanol. Y por otro lado, cuando estas señales están ausentes durante la fase de prueba no se presenta el desarrollo de la tolerancia cruzada al etanol (Crowel, Hinson \& Siegel, 1981), más bien se observa el efecto ansiolítico.

\section{DISCUSIÓN GENERAL}

Los resultados del primer experimento demostraron el efecto ansiolítico de la administración aguda diazepam y el etanol con dosis de $1 \mathrm{mg} / \mathrm{kg}$ y $1.5 \mathrm{~g} /$ $\mathrm{kg}$ respectivamente. El efecto ansiolítico observado con ambas drogas fue equivalente en ambas medidas, porcentaje de entradas y tiempo de permanencia en los brazos abiertos y no se detectaron cambios en la actividad motora debido al tratamiento. Una vez demostrado el efecto ansiolítico, en el segundo experimento se obtuvo el desarrollo de la tolerancia al efecto ansiolítico del diazepam por administración crónica (20 días) evaluado en el LEC. Este efecto no fue dependiente del contexto, a pesar de que se observó una tendencia del grupo tratado crónicamente y evaluado en diferente contexto a mostrar un mayor porcentaje de entradas y permanencia en los brazos abier- 
tos, lo que sugiere un efecto ansiolítico. En el último experimento se demostró el desarrollo de tolerancia cruzada diazepam-etanol al efecto ansiolítico, y que también la tolerancia cruzada puede ser modulada por mecanismos asociativos.

Los resultados de estos experimentos, se analizan bajo los supuestos de que la tolerancia a las drogas se desarrolla si los estímulos que estuvieron presentes en el momento de la administración de la droga quedan asociados con los efectos inducidos por ésta. Posteriormente cuando la prueba se realiza ante los mismos estímulos, éstos predicen la presentación de la droga y sus efectos (Cepeda-Benito \& Tiffany, 1996). Esto coincide con los observado en el Experimento 3, donde el contexto presente fue un factor importante en el desarrollo de tolerancia cruzada, sin embargo en el Experimento 2 la presencia del contexto no fue determinante en el desarrollo de la tolerancia asociativa a pesar de que se observó una tendencia del incremento al efecto ansiolítico, debido quizás como se mencionó previamente a la dosis empleada. Previamente reportamos que la administración de midazolam produjo tolerancia farmacológica y asociativa solamente cuando se utilizaron estímulos configuracionales y no estímulos discretos (Cruz-Morales \& Cedillo-lldefonso, 2008).

Estos resultados indican que se llevó a cabo una asociación entre señales y los efectos de la droga durante la inducción de ésta. En otras palabras la tolerancia fue dependiente del aprendizaje a todas las señales presentes durante las administraciones de la droga lo cual esta de acuerdo con los hallazgos de Baker y Tiffany (1985) y de Poulos y Cappell (1991). Se concluye que la tolerancia puede explicarse por mecanismos asociativos para la presencia o ausencia de la tolerancia, lo que contribuye al conocimiento y comprensión de mecanismos de aprendizaje del fenómeno de tolerancia como una explicación alternativa a la de los modelos farmacológicos en el desarrollo de la tolerancia a las asociaciones de la droga-ambiente, y que esta no es simplemente una alternativa a las interpretaciones tradicionales, más que esto, el modelo de condicionamiento es complementario a la explicación a otro nivel de otros puntos de vista de la presencia o ausencia de la tolerancia. Por último, se sugiere revisar la dosis administrada, para obtener resultados más robustos del efecto ansiolítico del diazepam.

\section{REFERENCIAS}

Baker, T. B. \& Tiffany, S. T. (1985). Morphine tolerance as habituation. Psychological Review, 92, 78-108.

Begg, D. P., Hallman, K. T. \& Norman, T. R. (2005). Attenuation of benzodiazepine withdrawal anxiety in the rat by serotonin antagonists. Behavioral Brain Research, 
$161,286-290$.

Bell R. L., Olson R. D. \& Vaccarino L. A. (1998). Tolerance to ethanol analgesia is not accompanied by cross-tolerance to morphine analgesia in rats. Pharmacology Biochemistry and Behavior, 59, 123-127.

Bertoglio, L. J. \& Carobrez A. P. (2002). Anxiolytic effects of ethanol and phenobarbital are abolished in test-experienced rats submitted to the elevated plus maze. Pharmacology Biochemistry and Behavior, 73, 963-969.

Caggiula, A. R., Epstein, L. H., Antelman, S. M., Taylor, S. S., Perkins, K., Knopf, S., \& Stiller, R. (1991). Conditioned tolerance to the anorectic effect and corticosterone-elevating effects of nicotine. Pharmacology Biochemistry and Behavior, 40, 53-59.

Cepeda-Benito, A., \& Tiffany S. T. (1996). Role of drugs-administration cues in the associative control of morphine tolerance in the rat. Psychopharmacology, 122, 312-316.

Cepeda-Benito, A., Tiffany, S. T., \& Cox L. S. (1999). Context-specific morphine tolerance on the paw-pressure and tail-shock vocalization test: Evidence of associative tolerance without conditioned compensatory responding. Psychopharmacology, $145,426-432$.

Chan, K. A., Langan, C. M., Leong, W. F., Schanley, L. D., \& Penetrante, L. M. (1988). Does chronic ethanol intake confer full cross-tolerance to chlordiazepoxide? Pharmacology Biochemistry and Behavior, 30, 385-389.

Cox, L. S., \& Tiffany, S. T. (1997). Associative and nonassociative tolerance: The effects of dose and interdose interval. Pharmacology Biochemistry and Behavior, $57,31-36$.

Crowell, C. R., Hinson, R. E., \& Siegel S. (1981). The role of conditional drug responses in tolerance to the hypothermic effects of ethanol. Psychopharmacology, 73, 51-54.

Cruz-Morales, S. E. \& Cedillo-Ildefonso, B. (en prensa). Tolerance to the anxiolytic effects of benzodiazepines. En: Méndez Ubach, M. \& Mondragón-Ceballos, R. (Eds.) Neural Mechanisms of Action of Drugs of Abuse and Natural Reinforcers. Research Signpost: Kerala, Índia.

de Angelis, L. \& File, S. E. (19979). Acute and chronic effects off three benzodiazepines in the social interaction anxiety test in mice. Psychopharmacology, 64, 127-129.

Fantino, E. (2001). Context: a central concept. Behavioral Processes, 54, 95-110.

Fernandes, C., \& File, S. E. (1999). Dizocilpine does not prevent the development of tolerance to the anxiolytic effects of diazepam in rats. Brain Research, 815, 431434.

File, S. E. (1982). Development and retention of tolerance to the sedative effects of chlordiazepoxide: Role of apparatus cues. European Journal Pharmacology, 81, 637-643.

File, S. E. (1985). Tolerance to the behavioral actions of benzodiazepines. Neuroscience \& Behavioral Reviews, 9, 1113-1121.

File, S. E., (1997). Anxiolytic action of a neurokinin1 receptor antagonist in the social interaction test. Pharmacology Biochemistry and Behavior, 58, 747-752.

File, S. E., Baldwin H. A, Aranko, K. (1987) Anxiogenic effects in benzodiazepine with- 
drawal are linked to the development of tolerance. Brain Research Bulletin, 19, 607-610.

Greeley, J. \& Cappell, H. (1985). Associative control of tolerance to the sedative and hypothermic effects of chlordiazepoxide. Psychopharmacology, 86, 487-493.

Harro, J., Lang, A. \& Vasar, E. (1990). Long-term diazepam treatment produces changes in cholecystokinin receptor binding in rat brain. European Journal Pharmacology, 180, 77-83.

Hijzen, T. H., Woudenberg, F. \& Slangen, J. L. (1990). The long-term effects of diazepam and pentylelenetetrazol on potentiated startle response. Pharmacology Biochemistry and Behavior, 36, 35-38.

Irvine, E. E., Cheeta, S., Lovelock, C. \& File, E. S. (2001). Tolerance to midazolam's anxiolytic effects after short-term nicotine treatment. Neuropharmacoloy, 40, 710716.

Kalynchuk, E. L., Kim, K. C., Pinel, J. P., \& Kippin, E. T. (1994). Effect of an ascending dose regimen on the development of tolerance to the anticonvulsant effect of diazepam. Behavioral Neuroscience, 108, 213-216.

Khanna, J. M., Kalant, H., Weiner, J., \& Shah, G. (1992). Rapid tolerance and crosstolerance as predictors of chronic tolerance and cross tolerance. Pharmacology Biochemistry and Behavior, 41, 355-360.

Khanna, J. M., Mihic, S. J., Weiner, J., Shah, G., Wu, P. H., \& Kalant, H. (1992). Differential inhibition by NMDA antagonists of rapid tolerance to, and cross-tolerance between, ethanol and chlordiazepoxide. Brain Research, 574, 251-256.

King, D. A., Bouton, M. E. \& Musty, R. E. (1987). Associative control of tolerance to the sedative effects of a short-acting benzodiazepine. Behavioral Neuroscience, 101, 104-114.

Le, A. D., Khanna, J. M., \& Kalant, H. (1987). Role of Pavlovian conditioning in the development of tolerance and cross-tolerance to hypothermic effect of ethanol and hydralazine. Psychopharmacology, 92, 210-214.

Le, D. A., Poulos, C. X. . \& Cappell, H. (1979). Conditional tolerance to the hypothermic effect of alcohol. Science, 206, 1109-1110.

Margules, D. L. \& Stein, L. (1968). Increase of "antianxiety" activity and tolerance of behavioral depression during administration of oxazepam. Psychopharmacologia, 13, 74-80.

Marin, H. R., Pérez, F. M., Duero, G. D., \& Ramírez, A.O. (1999). Preexposure to drug administration context blocks the development of tolerance to sedative effects of diazepam. Pharmacology Biochemistry and Behavior, 64, 473-477.

McKim, A. W. (1986). Drugs and Behavior. An Introduction to Behavioral Pharmacology. Nueva Jersey: Prentice-Hall.

O’ Brien, C. P. (1996). Adicción y Abuso de Sustancias. En Goodman, L. \& Gilman, G. L. (Eds.). Las Bases Farmacológicas de la Terapéutica (pp. 598-599). México: MacGraw-Hill.

Pavlov, I. P. (1927). Conditioned Reflexes. Londres: Oxford University Press.

Pérez, M. F., Maglio, L. E., Marchesini, G. R., Molina, J. C., \& Ramírez, O. A. (2002). Environmental changes modify the expression of diazepam withdrawal. Behavioral Brain Research, 136, 75-81.

Poulos, C. X., \& Cappell, H. (1991). Homeostatic theory of drug tolerance: a general 
model of physiological adaptation. Psychological Review, 98, 390-408.

Poulos, C. X., Hunt, T., \& Cappell, H. (1988). Tolerance to morphine analgesia is reduced by the novel addition of omission to an alcohol cue. Psychopharmacology (Berlin), 94, 412-416.

Rescorla, R. A., \& Wagner, A. R. (1972). A theory of pavlovian conditioning variations in the effectiveness of reinforcement and nonreinforcement. En A. H. Black \& Prokasy (Eds.), Classical Conditioning: Current Research and Theory (pp 64-69). Nueva York: Appleton-Century-Crofts.

Rodgers, R. J. \& Shepherd, J. K. (1993) Influence of prior maze experience on behaviour and response to diazepam in the elevated plus-maze and light/dark tests of anxiety in mice. Psychopharmacology (Berl), 113, 237-242.

Rosenberg, H. C. (1995) Differential expression of benzodiazepine anticonvulsant cross-tolerance according to time following flurazepam or diazepam treatment. Pharmacology Biochemistry and Behavior, 51, 363-374.

Schmitt, U., Luddens, H., Hiemke, C. (2001). Behavioral analysis indicates benzodiazepine-tolerance mediated by the benzodiazepine binding-site at the GABAareceptor. Progress in Neuro-Psychopharmacology and Biological Psychiatry, 25, $1145-1160$.

Siegel, S. (1975). Evidence from rats that morphine tolerance is a learned response. Journal of Comparative and Physiological Psychology, 89, 498-506.

Siegel, S. (1985). Drug anticipatory response in animals, En L.White, B. Tursky, and G. Schwartz (Eds.). In Placebo: Theory Research and Mechanisms (pp. 288305). Nueva York: Guilford Press.

Siegel, S. (1989). Pharmacological conditioning and drug effects. En M. W. EmmettOglesby, \& A. J. Goudie (Eds.). Tolerance and Sensitization to Psychoactive Drugs, Clifton, NJ: Human Press.

Siegel, S., \& Ramos, B. M. (2002) Applying laboratory research: drug anticipation and the treatment of drug addiction. Experimental and Clinical Psychopharmacology, $10,162-183$.

Stephens, D. N., \& Schneider, H. H. (1985). Tolerance to the benzodiazepine diazepam in an animal model of anxiolytic activity. Psychopharmacology (Berl), 87, 322-327.

Thompson, T. T., \& Boren, J. J. (1983). Farmacología conductual operante. En W. K Honig \& J. E. R. Staddon (Eds.), Manual de conducta operante (pp. 110-119). México: Trillas.

Treit, D. (1985a). Evidence that tolerance develops to the anxiolytic effect of diazepam in rats. Pharmacology Biochemistry and Behavior, 22, 383-387.

Treit, D. (1985b). The inhibitory effect of diazepam on defensive burying: Anxiolytic vs. analgesic effects. Pharmacology Biochemistry and Behavior, 22, 47-52.

Vellucci, S. V., \& File, S. E. (1979). Chlordiazepoxide loses its anxiolytic action with long-term treatment. Psychopharmacology, 62, 61-65. 